\title{
Some Alcohol-Related Consequences for Women May Signal an Escape from Interpersonal Violence
}

\author{
William H. Zywiak¹, Carolina L. Haass-Koffler², Alan Olinsky1,3, Leonardo Cavicchio, \\ David J. Ciliberto ${ }^{5}$ \\ ${ }^{1}$ Mathematics Department, Bryant University, Smithfield, RI, USA \\ ${ }^{2}$ Center for Alcohol and Addiction Studies, Department of Psychiatry and Human Behavior, Alpert Medical School; Department \\ of Behavioral and Social Sciences, School of Public Health, Brown University, Providence, RI, USA \\ ${ }^{3}$ Information Systems and Analytics Department, Bryant University, Smithfield, RI, USA \\ ${ }^{4}$ ISO|Verisk Analytics, Jersey City, NJ, USA \\ ${ }^{5}$ History and Social Sciences Department, Bryant University, Smithfield, RI, USA \\ Email: wzywiak@bryant.edu
}

How to cite this paper: Zywiak, W. H., Haass-Koffler, C. L., Olinsky, A., Cavicchio, L., \& Ciliberto, D. J. (2021). Some Alcohol-Related Consequences for Women May Signal an Escape from Interpersonal Violence. Advances in Applied Sociology, 11, 177-184.

https://doi.org/10.4236/aasoci.2021.114013

Received: March 22, 2021

Accepted: April 13, 2021

Published: April 16, 2021

Copyright $\odot 2021$ by author(s) and Scientific Research Publishing Inc. This work is licensed under the Creative Commons Attribution-NonCommercial International License (CC BY-NC 4.0). http://creativecommons.org/licenses/by-nc/4.0/

\section{(c) (i) (8) Open Access}

\begin{abstract}
Alcohol-related negative consequences can be very costly and emotionally devastating for those with Alcohol Use Disorder and those around them. Such, consequences include driving under the influence motor vehicle accident fatalities, other accidental deaths, and cirrhosis. We investigate two main queries using the data from Project COMBINE (Anton et al., 2006) to determine which negative consequences are most frequent for women, and to determine whether there are differences across ethnicity (for women) on negative consequences. The research method is secondary data analysis. An interpretation of the results turns the typical perspective on its head: We posit that results suggest women are escaping from interpersonal violence, which is of course an excellent outcome.
\end{abstract}

\section{Keywords}

Alcohol, Consequences, Women, Women of Color

\section{Introduction}

Across twenty different substances of misuse, Nutt, King, Saulsbury, and Blakemore (2007) rank the social harm associated with alcohol misuse as third, surpassed only by the social harm associated with heroin and cocaine. Healthcare costs are ranked by Nutt et al. in an identical fashion. Devastating consequences 
such as driving under the influence motor vehicle accident fatalities, other accidental deaths, and cirrhosis come to mind. The Drinker Inventory of Consequences (DrInC) was developed by Miller, Tonigan, and Longabaugh (1995) to provide a comprehensive sampling of possible primarily temporary alcohol problems. Item content was informed by the clinical practice of William Miller. This measure has been used in the Relapse Replication and Extension Project (Connors, Maisto, \& Zywiak, 1996), Project MATCH (Project MATCH Research Group, 1997), and Project COMBINE (Anton et al., 2006) as a secondary outcome measure in alcoholism treatment outcome studies; the primary outcome measures being some operationalization of alcohol consumption. The latter study with an $\mathrm{N}=1383$ is the second largest (compared to the Project MATCH $\mathrm{N}$ of 1726) and the most recent of these three multisite studies.

Conducting secondary data analyses on the Project COMBINE DrInC outcome data, we sought to provide preliminary answers to a number of questions.

1) Which negative consequences are most frequent for women?

2) Are there differences across ethnicity (for women) on negative consequences?

3) Are there differences across age (linear and quadratic) for women of color and white women?

There are two main scoring algorithms of the DrInC: the original algorithm (Miller, Tonigan, \& Longabaugh, 1995) and a more recent one developed using confirmatory factor analysis (Kirouac \& Witkiewitz, 2018). The original algorithm was based on face validity, and includes five clinical subscales: Intrapersonal Consequences, Physical Consequences, Relationship Consequences, Social Responsibility Consequences, and Impulse Control Consequences. Connors, Maisto, and Zywiak (1996) demonstrated construct validity of the measure by showing that alcohol involvement/symptoms and coping skills were related to negative consequences to the same degree that these antecedent variables were related to the two standard drinking outcome measures: percent days abstinent and drinks per drinking day. Miller, Tonigan, and Longabaugh (1995) provided empirical evidence for sound internal consistency and test-retest reliability of these scales. In contrast, to the original five clinical scales, Kirouac and Witkiewitz (2018) offer a three-factor solution, of six items (common consequences), twenty items (moderately common consequences), and nineteen items (rare consequences). Few analyses have compared the construct validity of the original and 2018 factors, and we present some of the first results to do this. We make a minor adjustment to the original scoring algorithm: previously scale scores for the DrInC have been calculated as totals. We re-normed these based on number of items, so that scales of different lengths (e.g., 7 versus 12 items) could be directly compared within subjects.

\section{Methods}

\subsection{Design}

The research design of Project COMBINE consisted of nine cells. There was a 2 
$\times 2 \times 2$ design yielding eight cells with acamprosate versus placebo crossed with naltrexone versus placebo crossed with a combined behavioral intervention (CBI) versus no intervention. Participants in all eight of these cells also received monitoring and reinforcement of medication adherence (medication management). There was an additional ninth cell used to assess a placebo effect in Project COMBINE, and this cell consisted of CBI with no pills (and therefore no medication management, Anton et al., 2006).

\subsection{Sample}

The sample consisted of 1383 clients. Inclusion criteria included current alcohol dependence, at least four and no more than 21 days of abstinence from alcohol. Volunteers were excluded if they had been abusing other substances in the last 90 days (except for nicotine and/or cannabis). The sample of 1383 included 428 (31\%) female clients and 321 (23\%) clients of color (Anton et al., 2006). Eight percent were Latino clients, $11 \%$ were Black clients, $77 \%$ were white clients. We focus on 301 women completing the DrInC at the one year follow-up.

\subsection{Measure}

The DrInC consists of 50 items. Respondents rate how much each consequence happened in the last 3 months, where $0=$ not at all, $1=$ a little, $2=$ somewhat, and 3 = very much. The original scoring algorithm of the DrInC yields five clinical scales (as well as a total scale). While the measure of negative consequences is administered before or at the beginning of treatment, we used the DrInC administered post-treatment rather than at baseline for a number of reasons. We avoided the confound of the length of the drinking career inflating endorsement of items. We avoided the confound of minimizing self-report due to a client's still coming to grips with an alcohol use disorder diagnosis. In other words, there are two competing influences to under-report and over-report consequences, which would increase error variance at the baseline administration of this measure. We chose the 1-year post-treatment assessment point in particular since that is a commonly used treatment outcome time point, and because of the large sample size that completed the $\mathrm{DrInC}$ at that time point. We therefore use the DrInC self-report pen and paper survey to assess post-treatment alcohol-related negative consequences (Table 1).

Table 1. Exemplar items for the DrInC scales.

\begin{tabular}{cl}
\hline Scale & \multicolumn{1}{c}{ Exemplar Items } \\
\hline Intrapersonal & I have felt bad about myself because of my drinking. \\
Physical & I have had a hangover after drinking. \\
Relationship & My family or friends have worried or complained about my drinking. \\
Social Responsibility & I have missed days of work or school because of my drinking. \\
Impulse Control & I have driven a motor vehicle after having three or more drinks. \\
\hline
\end{tabular}




\section{Results}

\subsection{Which Negative Consequences Are Most Frequent for Women? (Table 2)}

Paired Sample t-tests indicated each ranked DrInC scale was greater than the next lowest with all $p$ 's $<.001$, except for social responsibility versus impulse control with $\mathrm{t}(300)=2.78, p=.006$. Since we were going to use age as an independent variable in the regression analyses, we first conducted a preliminary analysis which showed that women of color in this study were younger [40.62 years $(\mathrm{SD}=10.31), \mathrm{t}(299)=4.03, p<.001]$ than white women $[46.27$ years $(\mathrm{SD}=$ 9.72)]. Women of color's age ranged from 21 to 61 years old. White women's age ranged from 24 to 72 .

\subsection{Are There Differences across Ethnicity (for Women) on Negative Consequences?}

Next, using means on the DrInC scale scores (administered 1-year after the 16 week medication schedule was completed) we compared women of color $(\mathrm{n}=$ $62)$ to white women $(n=239)$ using the DrInC. We found significant differences between the two groups on relationship alcohol-related negative consequences $[\mathrm{t}(73.1)=2.27, p=.026$, women of color: mean $=.57(\mathrm{SD}=.75)$ and white women: mean $=.35(\mathrm{SD}=.45)]$, social responsibility $[\mathrm{t}(81.9)=2.31, p=.023$, women of color: mean $=.47(\mathrm{SD}=.58)$ and white women: mean $=.28(\mathrm{SD}$ $=.46)]$, and impulse control scale scores $[\mathrm{t}(72.7)=2.64, p=.010$, women of color mean $=.38(\mathrm{SD}=.42)$ and white women mean $=.23(\mathrm{SD}=.25$, scales scores divided by number of items)]. See Figure 1 . Cognizant of the age differences in these two groups, these ethnicity differences were found to be robust, when we used stepwise regressions, with age entered in the first step, and ethnicity in the second step to assess associations with the DrInC scale scores, accounting for age [ $p$ 's for F-change (the second step of the regression) were .011 for ship, .052 for social responsibility, and .007 for impulse control alcohol-related negative consequences]. Using the Kirouac and Witkiewitz (2018) DrInC scoring algorithm, we found an ethnicity difference for rare negative consequences $[\mathrm{t}(71.8)=2.85, p=.006$, women of color mean $=.35(\mathrm{SD}=.42)$ and white women mean $=.19(\mathrm{SD}=.24)]$. Regression F change $p=.002$.

Table 2. Means and SDs for DrInC scales for women in project COMBINE $(\mathrm{N}=301)$.

\begin{tabular}{ccc}
\hline Scale & Mean & SD \\
\hline Intrapersonal & .87 & .86 \\
Physical & .53 & .57 \\
Relationship & .39 & .53 \\
Social Responsibility & .32 & .49 \\
Impulse Control & .27 & .30 \\
\hline
\end{tabular}




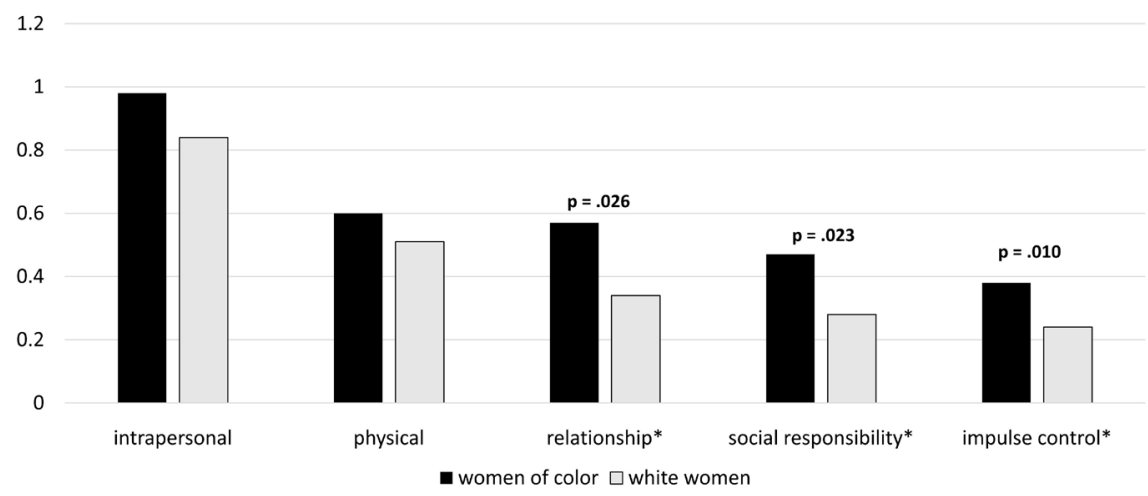

Figure 1. Negative consequences by Ethnicity ( ${ }^{\star}$ denotes ethnicity differences).

To help understand these findings, we ran similar t-tests comparing women of color to white women on responses on the individual items in the relationship, social responsibility, and impulse control alcohol-related scales. Group differences with $p$ 's $<.01$ consisted of item 21 "said harsh or cruel things to someone" $[\mathrm{t}(72.2)=2.72, p=.008$, women of color mean $=.66(\mathrm{SD}=.94)$ and white women mean $=.32(\mathrm{SD}=.55)]$, item 23 "gotten into a physical fight" $[\mathrm{t}(62.7)=$ $2.94, p=.005$, women of color mean $=.23(\mathrm{SD}=.56)$ and white women mean $=.02(\mathrm{SD}=.13)]$ and item 50 "I have broken things" $[\mathrm{t}(70.7)=2.97, p=.004$, women of color mean $=.40(\mathrm{SD}=.66)$ and white women mean $=.14(\mathrm{SD}=.36)]$. Women of color had higher means than white women. Item 22 is from the relationship scale and items 23 and 50 are from the impulse control scale.

\subsection{Are There Differences across Age (Linear and Quadratic) for Women of Color and White Women?}

Finally, for women of color we ran linear and quadratic regressions using age as an independent variable and DrInC scores as the dependent variables (using the Miller et al., 1995 and Kirouac and Witkiewitz, 2018 scoring rules). We then ran the same set of analyses for white women. None of the regressions were statistically significant for women of color. In contrast, for white women, two thirds of the original scales and one of the three Kirouac and Witkiewitz scales yielded statistically significant results. ("Model p" in Table 3, refers to the quadratic model if the $\mathrm{F}$ change was significant, the linear model, if this was not the case, and n.s. is indicated in the model p column, if both linear and quadratic models were not supported.) Two statistically significant though modest sized quadratic relationships were found. For white women, physical consequences peaked in the early 40 's and the total score peaked at 41 years old. If a quadratic relationship was statistically significant, we also verified that the F change was significant for age-squared entered in the second step of the regression, when age was entered in the first step of the regression.

\section{Discussion}

After The order of the most frequent negative consequences, from highest to 
Table 3. Significant linear and quadratic functions for white women.

\begin{tabular}{ccccccc}
\hline White Women, $\mathrm{n}=239$ & Model $p$ & Sig. F change & $\mathrm{R}^{2}$ & $\mathrm{a}\left(\beta_{2}\right)$ & $\mathrm{b}\left(\beta_{1}\right)$ & $\mathrm{c}\left(\beta_{0}\right)$ \\
\hline Intrapersonal & n.s. & & & & & \\
Physical Consequence & .013 & .025 & .036 & -.005 & .452 & -4.717 \\
Relationship & n.s. & & & & & \\
Social Responsibility & .002 & n.s. & .039 & $\mathrm{NA}$ & -.065 & 4.996 \\
Impulse Control & .002 & n.s. & .045 & $\mathrm{NA}$ & -.067 & 5.903 \\
Total & .025 & .047 & .031 & -.022 & 1.872 & -15.05 \\
Common & n.s. & & & & & \\
Moderately Common & n.s. & & & & & \\
Rare & .001 & n.s. & .047 & NA & -.005 & .438 \\
\hline
\end{tabular}

lowest was: intrapersonal, physical, relationship, social responsibility, and impulse control. Ethnic differences were found for the last three. Differences across age were found for white women, but not women of color. For white women, social responsibility consequences, impulse control consequences, and rare consequences decreased with age, suggesting prevention and treatment efforts focusing on younger women would be useful. While the R-squares are small, given that negative consequences can reverberate across a life, and the lives of those around one, these findings are important. (Analyses not presented here indicate that consequences vary with age for men of color and white men.) The null findings for age for women of color may be due to the smaller sample size ( $\mathrm{n}=$ 62) and/or chaotic surroundings of women of color with an alcohol diagnosis. In contrast to, Kirouac and Witkiewicz (2018), these results support the usefulness of the original DrInC scales (when normed for number of items in the scale).

In clinical practice, the DrInC might be used to flesh out the pros and cons of drinking versus abstinence (Marlatt \& Gordon, 1985). Clients rating intrapersonal and physical consequences higher than relationship, social responsibility, and impulse control, may reflect that clients are more willing to admit adverse consequences that do not seem to directly impinge on others. One possible avenue of future clinical work and or research might have partners rate the client on the DrInC items, with ratings compared. Indeed, collateral forms are included in the original DrInC manual (Miller, Tonigan, \& Longabaugh, 1995) as preliminary measures. A further modification of the DrInC might ask clients to rate how much a negative consequence bothers them, along the line of the Life Events Scale (Bailey, Koepsell, \& Belcher, 1984) $(-3=$ extremely negative, $-2=$ moderately negative, $-1=$ somewhat negative, $0=$ no effect, $+1=$ slightly, $+2=$ moderately, $+3=$ extremely positive). Thinking more broadly, alcohol treatment outcome studies should include more robust measures of socio-economic status, which may explain in part, the findings in this paper.

The original DrInC items were developed from the clinical practice of William Miller, which consisted of primarily male clients. It may be useful to develop ad- 
ditional items from clinicians and doctors that treat women and women of color, and or open-ended interviews with these clients. Conjecturing what may be going on for the clients, "saying harsh and cruel things to someone," "breaking things," and "getting into a physical fight" may reflect getting out of an abusive relationship (Davison, 2007; Malley-Morrison \& Hines, 2007). In other words, these consequences might be terrible for male clients or from a male perspective, but could be the beginning of a much better life for female clients from a feminist perspective (Hunt, Frank, \& Maloney, 2015; Peralta \& Jauk, 2011). Alcohol treatment outcome studies in the future might include the Dyadic Adjustment Scale (Spanier, 1988) and assess living situation and marital status longitudinally to test this hypothesis. This interpretation of these results is supported by Caetano, Cunradi, Clark, \& Schafer (2000) who found that intimate partner violence victim rates were higher for women of color than white women. It is important to note that we are not suggesting that partners of color are more likely to be violent, than white partners, since partner ethnicity was not assessed in Project COMBINE. Finally, other researchers (Merrill, Read, \& Barnett, 2013) have noted that while researchers may consider an alcohol-related consequence to be negative, this view may not always be shared by the actual survey respondents.

\section{Conclusion}

Results indicate that the most common alcohol-related negative consequences for women are (in order from most common to least common) intrapersonal, physical, relationship, social responsibility, and impulse control alcohol-related negative consequences. Relationship consequences, social responsibility consequences, and impulse control consequences were more pronounced for women of color than white women. These differences may be accounted for by fewer financial and social supports. Consequences varied by age for white women, but no for women of color, suggesting that alcohol use may affect women of color in more of a chaotic method compared to alcohol use for white women. Finally, item level analysis suggests that what looks like alcohol-related negative consequences for some women, may actually be a healthy escape from an abusive relationship.

\section{Conflicts of Interest}

The authors declare no conflicts of interest regarding the publication of this paper.

\section{References}

Anton, R. F., O’Malley, S. S., Ciraulo, D. A., Cisler, R. A., Couper, D., Donovan D. M. et al. (2006). Combined Pharmacotherapies and Behavioral Interventions for Alcohol Dependence: The COMBINE Study: A Randomized Trial. Journal of the American Medical Association, 295, 2003-2017. https://doi.org/10.1001/jama.295.17.2003

Bailey, G. A., Koepsell, T. D., \& Belcher, D. W. (1984). Reliability of Two Measures of Life Stress among Outpatients at a Veterans Hospital. American Journal of Public Health, 
74, 723-724. https://doi.org/10.2105/AJPH.74.7.723

Caetano, R., Cunradi, C. B., Clark, C. L., \& Schafer, J. (2000). Intimate Partner Violence and Drinking Patterns among White, Black, and Hispanic Couples in the U.S. Journal of Substance Abuse, 11, 123-138. https://doi.org/10.1016/S0899-3289(00)00015-8

Connors, G. J., Maisto, S. A., \& Zywiak, W. H. (1996). Understanding Relapse in the Broader Context of Post-Treatment Functioning. Addiction, 91, 173-189.

Davison, J. (2007). Alcohol Misuse: Contributor to and Consequence of Violence against Women. Diversity in Health and Social Care, 4, 137-148.

Hunt, G., Frank, V. A., \& Moloney, M. (2015). Introduction: Rethinking Gender with Alcohol and Drug Research. Substance Use and Misuse, 50, 685-692. https://doi.org/10.3109/10826084.2015.978635

Kirouac, M. \& Witkiewicz, K. (2018). Revisiting the Drinker Inventory of Consequences: An Extensive Evaluation of Psychometric Properties in Two Alcohol Clinical Trials. Psychology of Addictive Behaviors, 32, 52-63. https://doi.org/10.1037/adb0000344

Malley-Morrison, K. \& Hines, D. A. (2007). Attending to the Role of Race/Ethnicity in Family Violence Research. Journal of Interpersonal Violence, 22, 943-972. https://doi.org/10.1177/0886260507303060

Marlatt, G. A. \& Gordon, J. R. (1985). Relapse Prevention: Maintenance Strategies in the Treatment of Addictive Behaviors. New York, NY: Guilford.

Merrill, J. E., Read, J. P., \& Barnett, N. P. (2013). The Way one Thinks Affects the Way One Drinks: Subjective Evaluations of Alcohol Consequences Predict Subsequent Changes in Drinking Behavior. Psychology of Addictive Behaviors, 27, 42-51. https://doi.org/10.1037/a0029898

Miller, W. R., Tonigan, J. S., \& Longabaugh, R. (1995). Drinker Inventory of Consequences (Vol. 4). Project MATCH Monograph Series. Rockville, MD: APA PsycTests. https://doi.org/10.1037/t03945-000

Nutt, D., King, L. A., Saulsbury, W., \& Blakemore, C. (2007). Development of a Rational Scale to Assess the Harm of Drugs of Potential Misuse. Lancet, 369, 1047-1053. https://doi.org/10.1016/S0140-6736(07)60464-4

Peralta, R. L. \& Jauk, D. (2011). A Brief Feminist Review and Critique of the Sociology of Alcohol-Use and Substance-Abuse Treatment Approaches. Sociology Compass, 5, 882-897. https://doi.org/10.1111/j.1751-9020.2011.00414.x

Project MATCH Group Research Group (1997). Matching Alcohol Treatments to Client Heterogeneity: Project MATCH Posttreatment Drinking Outcomes. Journal of Studies on Alcohol, 58, 7-29. https://doi.org/10.15288/jsa.1997.58.7

Spanier, G. B. (1988). Assessing the Strengths of the Dyadic Adjustment Scale. Journal of Family Psychology, 2, 92-94. https://doi.org/10.1037/h0080477 